\title{
Adiabatic passage and ensemble control of quantum systems
}

\author{
Z Leghtas ${ }^{1,4}$, A Sarlette ${ }^{2,3}$ and P Rouchon ${ }^{3}$ \\ ${ }^{1}$ INRIA Paris-Rocquencourt Domaine de Voluceau, BP105 78153 Le Chesnay Cedex, France \\ 2 Department of Electrical Engineering and Computer Science, University of Liège, 4000 Liège, Belgium \\ ${ }^{3}$ Mines-ParisTech, Centre Automatique et Systèmes, 60, boulevard Saint-Michel 75272 Paris Cedex, \\ France \\ E-mail: zaki.leghtas@inria.fr, alain.sarlette@ulg.ac.be and pierre.rouchon@mines-paristech.fr
}

Received 24 December 2010

Published 25 July 2011

Online at stacks.iop.org/JPhysB/44/154017

\begin{abstract}
This paper considers population transfer between eigenstates of a finite quantum ladder controlled by a classical electric field. Using an appropriate change of variables, we show that this setting can be set in the framework of adiabatic passage, which is known to facilitate ensemble control of quantum systems. Building on this insight, we present a mathematical proof of robustness for a control protocol—chirped pulse-practised by experimentalists to drive an ensemble of quantum systems from the ground state to the most excited state. We then propose new adiabatic control protocols using a single chirped and amplitude-shaped pulse, to robustly perform any permutation of eigenstate populations, on an ensemble of systems with unknown coupling strengths. These adiabatic control protocols are illustrated by simulations on a four-level ladder.
\end{abstract}

(Some figures in this article are in colour only in the electronic version)

\section{Introduction}

Population transfer from the eigenstate $k$ to the eigenstate $l$ of a quantum system refers to finding a control input such that the projection of the final system state on the eigenstate $l$ of the free Hamiltonian has the same norm as the projection of the initial system state on the eigenstate $k$. Applications of population transfer range from population inversion [1], where $k$ and $l$ are the lowest and highest energy eigenstates, respectively, to quantum information processing [2-4], where logic gates would (selectively) permute the populations of several eigenstates. In many applications, including those mentioned, relative insensitivity to variations in system parameters is important for robustness issues.

In this paper, we show how control inputs designed on the basis of adiabatic passage can implement any given permutation of eigenstate populations for a finite anharmonic quantum ladder. The controls we use are chirped pulses [5] with appropriately modulated amplitudes and exploit the idea of eigenvalue crossing [6]. The ladder consists of a free Hamiltonian with approximately equidistant eigenvalues

\footnotetext{
4 Author to whom any correspondence should be addressed.
}

and where the control input couples eigenstates associated with consecutive eigenvalues. We show that any control field satisfying a set of key properties achieves our target population transfer independently of the values of dipole moments coupling consecutive levels of the ladder, which is a striking robustness feature. This is a major difference with respect to early non-adiabatic approaches to molecular ladder dissociation using chirped pulses [5]. Adiabatic passage through eigenvalue crossings has also very recently been used to prove approximate controllability in finite time of an infinite dimensional quantum system [7].

In this sense, we achieve a specific form of ensemble control. Ensemble control in its most general form wants the same input to drive an ensemble of systems, with different values of some parameter $p$, from a given $p$-dependent initial state to a given $p$-dependent final state ([8], definition 1). Currently, solutions to this general problem are essentially restricted to two-level systems, achieving approximate ensemble control in finite time and exact ensemble control in infinite time [8-10]. They rely on accurate knowledge of laser-system coupling strengths and accurately tailored inputs, involving e.g. exact instantaneous ' $\pi$-amplitude impulses'. In our setting, system parameters need not be exactly known 
and the input must only satisfy a few key properties. In turn, regarding initial-to-final-state transformations, we are limited to population permutations (with arbitrary relative phases between components of different eigenstates) that are constant as a function of system parameters. Driving an ensemble of two-level systems from a common initial to a common final state has also been much studied in the NMR context, e.g. with geometric methods [9].

Adiabatic passage is a control strategy that builds on the adiabatic evolution property: a system state initially close to an eigenstate of a time-varying Hamiltonian $H(t)$ approximately follows the time-varying eigenstate of $H(t)$ if it varies slowly enough; the slower $H(t)$ varies, the better the adiabatic approximation is. A thorough formal study of adiabatic evolution can be found in [11-13], on which we build the proofs of our results. Adiabatic evolution has been standard since the early days of quantum mechanics [14], e.g. when interpreting system evolution in terms of 'avoided eigenvalue crossings'. In a ladder control context, population inversion in two-level systems by a 'chirped' pulse-where frequencies of a Gaussian laser pulse are spread out in timeis known by experimentalists and theoretically explained in the adiabatic framework [15]. This is the most basic case of our control, section 3 with $N=2$. Many experimentalists have then focused on multiple-laser techniques, individually addressing pairwise couplings in an $\mathrm{N}$-level system; this includes stimulated Raman adiabatic passage (STIRAP), see e.g. [16-18]. For $N$-level ladder systems specifically, the possibility of population transfer from the lowest to the highest energy eigenstates with a single chirped laser pulse has been recognized and exploited in 'adiabatic rapid passage' experiments [1, 18-20]. An analysis of $N$-level adiabatic molecular dissociation with chirped pulses is given in [21] based on the Floquet representation. In the present paper we provide a simple mathematical proof of population inversion with avoided crossings (gap condition) based on Favard's theorem [22] and on the roots of orthogonal polynomials [23], and extend the framework by adding amplitude control to perform not only population inversion but all different permutations of free Hamiltonian eigenstates.

The paper is organized as follows. Section 3 gives the formal statement and section 6 the proof for $N$-level population inversion with 'adiabatic rapid passage', actually proving how the initial population of level $k$ is finally transferred to level $N-k-1$ in adiabatic approximation. The key point for using adiabatic passage is a change of frame that depends on time-varying control input phase; it is detailed in section 2 after a formal description of the ladder system. The proof then applies the standard 'adiabatic theorem with spectral gap condition', where time-varying eigenvalues are shown to remain separated for all times. The inversion is insensitive to exact energy values of the individual levels in the ladder. Section 4 proposes adiabatic control inputs to transfer population between two arbitrary eigenstates. It requires the control field to vanish at specific times which depend on (some) energy levels of the anharmonic ladder, such that we select a pair of time-varying eigenvalues to cross. System evolution is then ruled by the 'adiabatic theorem without spectral gap condition'. A complementary study of system behaviour in the neighbourhood of two crossing eigenvalues and valid for more general systems than ladder ones can be found in [24]. We again provide a formal proof of the control's effect and highlight its ensemble/robustness features in section 6 . Section 5 finally shows how any permutation of eigenstate populations can be achieved in this adiabatic passage framework. Each control protocol is illustrated by a simulation at the end of the corresponding section.

Notation. We use the Dirac bra-ket notations: $|\psi\rangle \in \mathbb{C}^{N}$ denotes a complex vector, $\langle\psi|=| \psi\rangle^{\dagger}$ is its Hermitian transpose and $\langle. \mid\rangle:. \mathbb{C}^{N} \times \mathbb{C}^{N} \rightarrow \mathbb{C}:\left(\left|\psi_{1}\right\rangle,\left|\psi_{2}\right\rangle\right) \rightarrow$ $\left\langle\psi_{1} \mid \psi_{2}\right\rangle=\left\langle\psi_{1}|| \psi_{2}\right\rangle$ is the Hermitian scalar product. For $z \in \mathbb{C}$ we denote its real part as $\Re(z)$ and its conjugate as $z^{*}$. $\mathcal{H}_{N}$ is the set of $N \times N$ Hermitian matrices, where $N \in \mathbb{N}$. We denote $I$ as the $N \times N$ identity matrix. For any matrix $A \in \mathbb{C}^{N \times N}$, we denote its Frobenius (or HilbertSchmidt) norm as $\|A\|=\sqrt{\operatorname{tr} A^{\dagger} A}$ where tr denotes trace. For $H \in \mathcal{H}_{N}$, it holds $\|H\|=\sqrt{\sum_{i=0}^{N-1} \lambda_{i}^{2}}$ where $\lambda_{0}, \ldots, \lambda_{N-1}$ are the (real) eigenvalues of $H$. For $H \in \mathcal{H}_{N}$ and $\lambda$ an eigenvalue of $H$, we denote by $P_{\lambda} \in \mathcal{H}_{N}$ the orthogonal projector on the eigenspace of $H$ associated with the eigenvalue $\lambda$. If $H$ has $M$ distinct eigenvalues $\left\{\lambda_{0}, \ldots, \lambda_{M-1}\right\}$, with $M \leqslant N$, then $H=\sum_{k=0}^{M-1} \lambda_{k} P_{\lambda_{k}}$ is the spectral decomposition of $H$. If $M=N$, then $H$ is called non-degenerate and each $P_{\lambda_{k}}$ is a rank-one projector. When $M<N$ we say that $H$ is degenerate; then some $P_{\lambda_{k}}$ have rank larger than 1 .

$\mathbb{S}^{1}$ denotes the unit circle equivalent to $\mathbb{R}$ modulo $2 \pi$. For $J$ an interval of $\mathbb{R}$, the derivative of a differentiable function $f: J \rightarrow \mathbb{S}^{1}$ is a function from $J$ to $\mathbb{R}$. For all $n \in \mathbb{N}$, we denote by $\mathcal{C}^{n}(J, K)$ the set of $n$ times continuously differentiable functions from $J$ to $K$, where $J$ is an interval of $\mathbb{R}$ and $K$ is an interval of $\mathbb{R}$ or $\mathbb{S}^{1}$. A multi-component function is $n$ times continuously differentiable, e.g. $H(s) \in \mathcal{C}^{n}\left(J, \mathcal{H}_{N}\right)$, if all its components belong to $\mathcal{C}^{n}(J, K)$. For $f \in \mathcal{C}^{1}\left(J, K \subseteq \mathbb{R}^{n}\right)$, we note $f^{\prime}(y) \in \mathcal{C}^{0}\left(J, \mathbb{R}^{n}\right)$ the value at $y \in J$ of the derivative of $f . \mathbb{R}_{>0}$ is the set of strictly positive real numbers; we use analogue notation with $\geqslant, \leqslant$ or $<. \quad \mathbb{N}_{a}^{b}$ is the set of integers from $a$ to $b$, both boundaries included. When writing $c_{0}, \ldots, c_{N-1} \in \mathcal{S}$ we mean that $c_{k}$ belongs to the set $\mathcal{S}$ for each $k \in \mathbb{N}_{0}^{N-1}$. Infimum and supremum of a set are denoted by inf and sup respectively.

\section{Problem setting}

\subsection{Standard formulation}

Consider a quantum system with wavefunction $|\psi\rangle \in$ $\mathbb{C}^{N},\langle\psi \mid \psi\rangle=1, N \in \mathbb{N}$, whose dynamics is governed by the Schrödinger equation (with $\hbar=1$ )

$$
\mathrm{i} \frac{\mathrm{d}}{\mathrm{d} t}|\psi(t)\rangle=\left(H_{0}+u(t) H_{1}\right)|\psi(t)\rangle .
$$

The Hamiltonians $H_{0} \in \mathcal{H}_{N}$ and $H_{1} \in \mathcal{H}_{N}$ respectively characterize free and control-induced evolution, $u(t)$ being a real scalar control. In this paper, we consider a quantum 
ladder for which the Hamiltonians, in the eigenbasis $\{|0\rangle, \ldots$, $|N-1\rangle\}$ of $H_{0}$, take the form

$$
\begin{gathered}
H_{0}=\sum_{k=0}^{N-1} k\left(\omega_{0}+\Delta_{k}\right)|k\rangle\langle k| \\
H_{1}=\sum_{k=0}^{N-2} \mu_{k}(|k\rangle\langle k+1|+| k+1\rangle\langle k|),
\end{gathered}
$$

with $\omega_{0} \in \mathbb{R}_{>0} ; \Delta_{0}, \ldots, \Delta_{N-1} \in \mathbb{R} ;$ and $\mu_{0}, \ldots, \mu_{N-2} \in$ $\mathbb{R}_{>0}$. We assume that the system (1) features two very different orders of magnitude:

$$
|| u(t) H_{1}|| \approx\left|\Delta_{k}\right| \ll \omega_{0} \quad \text { for all } k \text { and all } t .
$$

Physically, $H_{0}$ is the free Hamiltonian of a quantum ladder with mean resonant frequency $\omega_{0}$ and anharmonicities $\Delta_{k}$. We call eigenstates $|0\rangle, \ldots,|N-1\rangle$ of $H_{0}$ the levels of the ladder. $H_{1}$ is the dipole moment matrix and models couplings between consecutive eigenstates; it is therefore tridiagonal with zero diagonal elements, and can be taken real positive and symmetric without loss of generality. Condition (4) expresses that control amplitude is relatively weak and that the ladder is close to a harmonic one, i.e. eigenvalues of $H_{0}$ associated with consecutive eigenstates are close to equidistant. This allows us to exploit resonant transitions between all consecutive eigenstates with control of carrier frequency $\omega_{0}$. We consider a typical such control with a small positive parameter $\varepsilon$ :

$$
\begin{gathered}
u(t)=2 \Re\left(\mathrm{e}^{\mathrm{i} \omega_{0} t} E(t)\right), \quad E(t)=A(\varepsilon t) \mathrm{e}^{\frac{\mathrm{i}}{\varepsilon} \theta(\varepsilon t)} \\
\text { with }\left\|\frac{\mathrm{d}}{\mathrm{d} t} E(t)\right\| \ll \omega_{0},
\end{gathered}
$$

where $A(t) \in \mathbb{R}$ and $\theta(t) \in \mathbb{S}^{1}$ for all $t \in \mathbb{R}_{\geqslant 0}$. The parameter $\varepsilon$ governs the rate of variations in the envelope $A(\varepsilon t)$ and frequency $\frac{\mathrm{d}}{\mathrm{d} t} \frac{1}{\varepsilon} \theta(\varepsilon t)=\theta^{\prime}(\varepsilon t)$ of $E(t)$; we show in the next sections how taking $\varepsilon$ small allows us to apply adiabatic passage properties. The slow but nonzero frequency variation is a key element for our control strategy. Physically, control fields like (5) are obtained e.g. by 'shaping' a single-laser pulse [25].

The rotating wave approximation (RWA), standard in quantum system modelling, consists in writing (1) with the change of variable $|\phi(t)\rangle=\left(\sum_{k=0}^{N-1} \mathrm{e}^{\mathrm{i} k \omega_{0} t}|k\rangle\langle k|\right)|\psi(t)\rangle$ and neglecting fast oscillating terms, to keep only those that vary at frequencies $\ll \omega_{0}$. It can be justified by averaging theory [26] thanks to inequalities (4), (6). Within this approximation, $|\phi\rangle$ follows the dynamics

$$
\mathrm{i} \frac{\mathrm{d}}{\mathrm{d} t}|\phi(t)\rangle=\left(\bar{H}_{I}+\widetilde{H}_{I}(t)\right)|\phi(t)\rangle
$$

where

$$
\begin{gathered}
\bar{H}_{I}=\sum_{k=0}^{N-1} k \Delta_{k}|k\rangle\langle k| \\
\widetilde{H}_{I}(t)=\sum_{k=0}^{N-2} \mu_{k}\left(E(t)|k\rangle\left\langle k+1\left|+E^{*}(t)\right| k+1\right\rangle\langle k|\right) .
\end{gathered}
$$

\subsection{Change of frame}

The Hamiltonian $\widetilde{H}_{I}(t)$ contains a control field whose phase $\frac{1}{\varepsilon} \theta(\varepsilon t)$ varies on timescales of order 1 . The key idea to apply adiabatic passage to the $N$-level system is an appropriate further change of frame on (7), such that all explicit time dependence in the resulting dynamics involves timescales of order $\varepsilon$. To this end, we extend the change of frame given in [15], section 4.6, for the two-level case and define $|\xi(t)\rangle=\sum_{k=0}^{N-1} \mathrm{e}^{k \frac{i}{\varepsilon} \theta(\varepsilon t)}|k\rangle\langle k|| \phi(t)\rangle$. Dynamics (7) becomes

$$
\mathrm{i} \frac{\mathrm{d}}{\mathrm{d} t}|\xi(t)\rangle=\left(H_{R}(\omega(\varepsilon t))+A(\varepsilon t) H_{1}\right)|\xi(t)\rangle
$$

with $\omega=\theta^{\prime}, H_{1}$ given by (3) and

$$
H_{R}(v)=\sum_{k=0}^{N-1} k\left(\Delta_{k}-v\right)|k\rangle\langle k| \quad \text { for all } v \in \mathbb{R} .
$$

Define the propagator $U^{\varepsilon}$ to be a time-dependent $N$ by $N$ unitary matrix such that the solution of (8) is given by $|\xi(t)\rangle=U^{\varepsilon}(t)|\xi(0)\rangle$ for all $t$ and for all $|\xi(0)\rangle$. Then $U^{\varepsilon}$ follows the dynamics

$$
\begin{gathered}
\mathrm{i} \varepsilon \frac{\mathrm{d}}{\mathrm{d} s} U^{\varepsilon}(s)=H(s) U^{\varepsilon}(s), \quad U^{\varepsilon}(0)=I \\
\text { with } \quad H(s)=H_{R}(\omega(s))+A(s) H_{1}
\end{gathered}
$$

in the timescale $s=\varepsilon t$. In the following, we study the system (10) for $s$ in the interval $[0,1]$ and with $A(s)$ and $\omega(s)$ as controls. Our goal is to achieve:

(a) Adiabatic approximate eigenstate permutations:

$$
\lim _{\varepsilon \rightarrow 0^{+}} \max _{k \in G} \| U^{\varepsilon}(1)|k\rangle\left\langle k\left|U^{\varepsilon}(1)^{\dagger}-\right| \sigma(k)\right\rangle\langle\sigma(k)| \|=0
$$

for given $G \subseteq \mathbb{N}_{0}^{N-1}$ and given permutation $\sigma$ of $(0, \ldots, N-1)$.

(b) Ensemble control: a single control $(A, \omega)$ achieves such eigenstate permutation on an ensemble of systems with different parameter values; the parameters are the dipole moments $\left(\mu_{0}, \ldots, \mu_{N-2}\right)$ and, in some cases, the anharmonicities $\left(\Delta_{0}, \ldots, \Delta_{N-1}\right)$.

(c) Robust control inputs: the above holds for any $(A, \omega)$ that satisfies a set of key properties.

Remark 1. Writing (12) in terms of $|k\rangle\langle k|$, the projector on the eigenspace $\{\beta|k\rangle: \beta \in \mathbb{C}\}$, expresses that the goal is really population transfer, i.e. we allow $U^{\varepsilon}(1)|k\rangle \approx \mathrm{e}^{\mathrm{i} \chi_{k}}|\sigma(k)\rangle$ with arbitrary phases $\chi_{k} \in \mathbb{S}^{1}$. Both frame changes-for RWA in section 2.1 and $\theta$-dependent in section 2.2-involve only phase changes on eigenstates. Therefore, for all $t$ and for all $|k\rangle$,

$$
\begin{aligned}
\||\psi(t)\rangle\langle\psi(t)|-| k\rangle\langle k| \| & =\||\phi(t)\rangle\langle\phi(t)|-| k\rangle\langle k| \| \\
& =\||\xi(t)\rangle\langle\xi(t)|-| k\rangle\langle k| \| .
\end{aligned}
$$




\section{Robust ensemble transfer from $|k\rangle$ to $|N-k-1\rangle$}

In this section we consider a control protocol—chirped pulse - used by physicists to drive a system from the lowest eigenspace, spanned by $|0\rangle$, to the highest eigenspace, spanned by $|N-1\rangle$, of the free Hamiltonian $H_{0}$ given in (2). In fact we prove that a general (robust) class of control inputs transfers population from the eigenstate $|k\rangle$ to the eigenstate $|N-k-1\rangle$, for all $k$, on an ensemble of systems with different values of parameters $\mu_{0}, \ldots, \mu_{N-2}$ (dipole moments) and $\Delta_{0}, \ldots, \Delta_{N-1}$ (anharmonicities).

The key requirements on the control are (i) to use a sufficiently chirped pulse-condition (b) in theorem 1and (ii) to avoid all eigenvalue crossings-condition (c) in theorem 1 .

\subsection{Transfer theorem}

For $k=0, \ldots, N-1$ let $\lambda_{k}^{R}(s)=\left\langle k\left|H_{R}(\omega(s))\right| k\right\rangle=$ $k\left(\Delta_{k}-\omega(s)\right)$, the eigenvalues of $H_{R}(\omega(s))$.

Theorem 1. For given $\Delta>0, \mu_{\max }>\mu_{\min }>0$, consider $\mathcal{S}$ an ensemble of systems of type (10) with $\mu_{j} \in\left[\mu_{\min }, \mu_{\max }\right]$ for all $j \in \mathbb{N}_{0}^{N-2}$ and $\Delta_{j} \in[-\Delta, \Delta]$ for all $j \in \mathbb{N}_{0}^{N-1}$. Take controls $A$ and $\omega$ with

(a) $A$ and $\omega \in \mathcal{C}^{2}([0,1], \mathbb{R})$,

(b) $\omega(0)$ and $\omega(1)$ are such that, for all systems in $\mathcal{S}$,

$$
\begin{gathered}
\lambda_{0}^{R}(0)<\cdots<\lambda_{N-1}^{R}(0) \text { and } \\
\lambda_{0}^{R}(1)>\cdots>\lambda_{N-1}^{R}(1),
\end{gathered}
$$

(c) $A(0)=A(1)=0$ and $A(s) \neq 0$ for $s \in] 0,1[$.

Then $\exists$ a constant $C>0$ such that, for all $\varepsilon>0$,

$\sup _{S} \| U^{\varepsilon}(1)|k\rangle\left\langle k\left|U^{\varepsilon}(1)^{\dagger}-\right| N-k-1\right\rangle\langle N-k-1| \| \leqslant C \varepsilon$. $\underset{k \in \mathbb{N}_{0}^{N-1}}{\mathcal{S}}$

The proof of this theorem is given in section 6 ; we there actually replace the simple condition (c) on $A$ by a more general condition: $\left(\mathrm{c}^{\prime}\right) A(0)=A(1)=0$ and $A(s) \neq 0$ for all $s \in \mathcal{I}^{\omega}(\mathcal{S})$, where

$\mathcal{I}^{\omega}(\mathcal{S})=\{s \in[0,1]:$

$H_{R}(\omega(s))$ is degenerate for some system $\left.\in \mathcal{S}\right\}$.

The argument is based on the facts that the system approximately follows eigenstates of $H(s)$ for small enough $\varepsilon$ (adiabatic theorem), eigenvalues of $H_{R}$ are inverted between $s=0$ and $s=1$ thanks to $\omega(s)$ (chirping) and nonzero $A(s)$ avoids all crossings for eigenvalues of $H(s)$ such that e.g. the initial highest-energy level $|N-1\rangle$ connects to the final highest-energy level $|0\rangle$ (see lemma 1 in section 6). Theorem 1 implies that for a given control satisfying the assumptions, taking $\varepsilon$ small enough allows us to invert the state populations of a whole ensemble of systems featuring different parameter values. The control inputs only need to satisfy a few weak conditions and are therefore robust to many perturbations. These insensitivity properties of the adiabatic passage protocol have long been recognized by experimentalists. They commonly use the following type of control, see e.g. [18].

Example 1. A function $\omega$ satisfying the inequalities (13) is e.g. $\omega(s)=\alpha\left(s-\frac{1}{2}\right)$, for a large enough positive $\alpha$; such $\omega$ is said to perform a frequency sweep. Except for the finite extension of time domain, such inputs are obtained by a 'chirped' Gaussian laser pulse, which takes the form $E(t)=E_{0} \int_{-\infty}^{+\infty} \mathrm{e}^{-\zeta^{2} \tau^{2}} \mathrm{e}^{\mathrm{i} \kappa \zeta^{2}} \mathrm{e}^{-\mathrm{i} \zeta t} \mathrm{~d} \zeta$ where $\kappa \neq 0$ characterizes chirping.

Theorem 1 still holds if inequality (13) is replaced by

$$
\lambda_{0}^{R}(0)>\cdots>\lambda_{N-1}^{R}(0) \text { and } \lambda_{0}^{R}(1)<\cdots<\lambda_{N-1}^{R}(1),
$$

i.e. the direction of the frequency sweep in example 1 can be inverted (taking a large enough negative $\alpha$ ). However, for a given system, choosing one inequality over the other may allow us to get a lower value for the constant $\mathrm{C}$ in theorem 1 . This brings a mathematical foundation to the experimental observations made e.g. in [20].

\subsection{Simulations}

We simulate the system (10) with a control satisfying assumptions (a), (b) and (c) of theorem 1. We consider a four-level quantum ladder (so $N=4$ ). We take $\varepsilon=10^{-2}$, $\Delta_{0}, \ldots, \Delta_{3} \in[-0.4,0.4]$ and $\mu_{0}, \mu_{1}, \mu_{2} \in\left[\mu_{\min }, \mu_{\max }\right]=$ $[1,5]$. The control is $\omega(s)=8\left(s-\frac{1}{2}\right)$ and $A(s)=s(1-s)$, represented in figure 1(a). Figure 1(b) shows how the eigenvalues of $H(s)$ (thick lines) avoid crossing. For the illustrated random choice of detunings, the eigenvalues of $H_{R}(\omega(s))$ (thin lines) are very close to concurrent between $s=0.5$ and $s=0.6$. This poses no problem for the adiabatic transfer from $|k\rangle$ to $|N-k-1\rangle$. The successful transfer is illustrated in figure $1(\mathrm{~d})$, which shows the squared norm of the projection of $U^{\varepsilon}(1)|k\rangle$ onto $|p\rangle$, for all pairs $(|k\rangle,|p\rangle)$ of eigenvectors of $H_{0}$; this is equivalent to the squared norm of the element on row $p$, column $k$ of the matrix $U^{\varepsilon}(1)$ that acts by left-multiplication on initial column vectors, for $U^{\varepsilon}(1)$ expressed in the basis $(|0\rangle, \ldots,|3\rangle)$. Figure 1(c) shows ensemble control on ten systems with different random values of $\Delta_{0}, \ldots, \Delta_{3}$ and $\mu_{0}, \mu_{1}, \mu_{2}$.

\section{Robust ensemble transfer from $|l\rangle$ to $|p\rangle$}

In this section we propose a new robust control protocol to drive a system from the eigenspace (of free Hamiltonian $H_{0}$ ) spanned by $|l\rangle$ to the eigenspace spanned by $|p\rangle$, for any given $l$ and $p$ in $\mathbb{N}_{0}^{N-1}$. The population transfer works on an ensemble of systems with different values of $\mu_{0}, \ldots, \mu_{N-2}$ (dipole moments), and for a general class of inputs where zero-crossings of $A(s)$ must be correlated with degeneracies of $H_{R}(\omega(s))$; the latter depend on $\omega(s)$ and (some of the) anharmonicities $\Delta_{0}, \ldots, \Delta_{N-1}$, which must hence be fixed. 
(a)

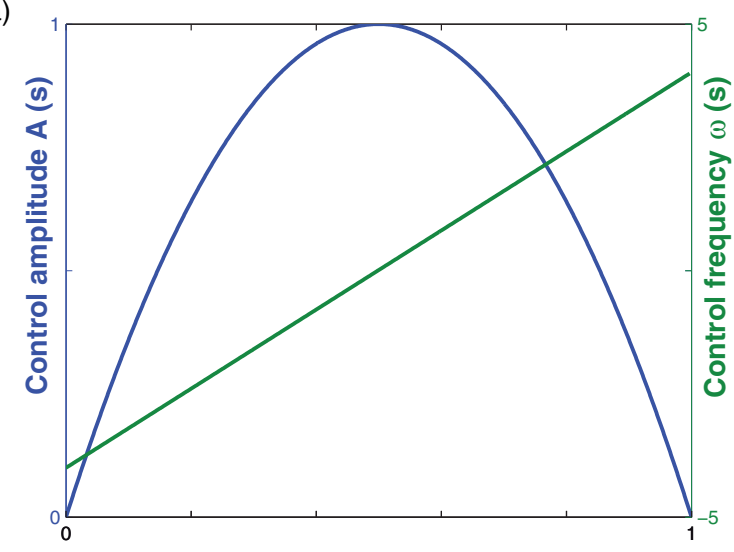

(c)

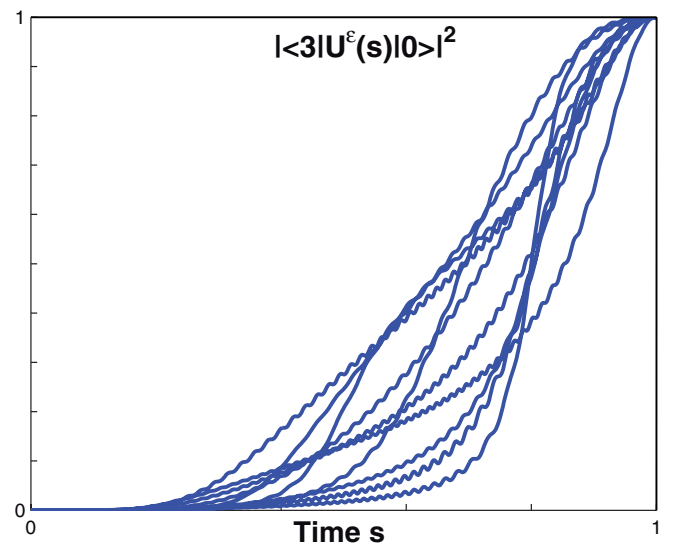

(b)

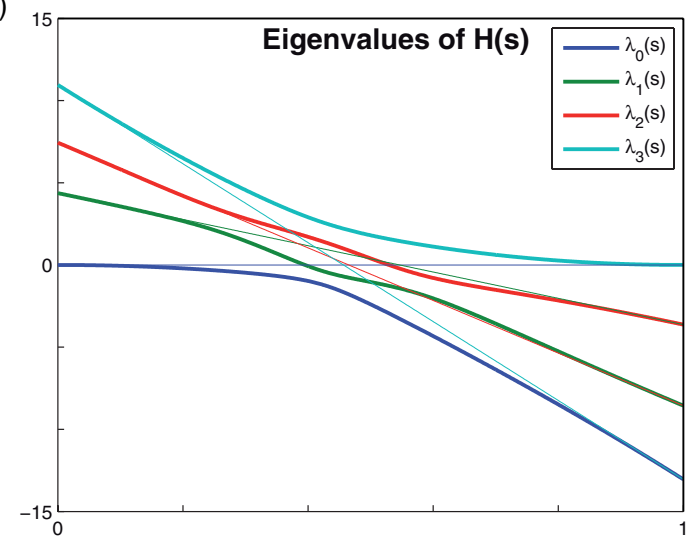

(d)

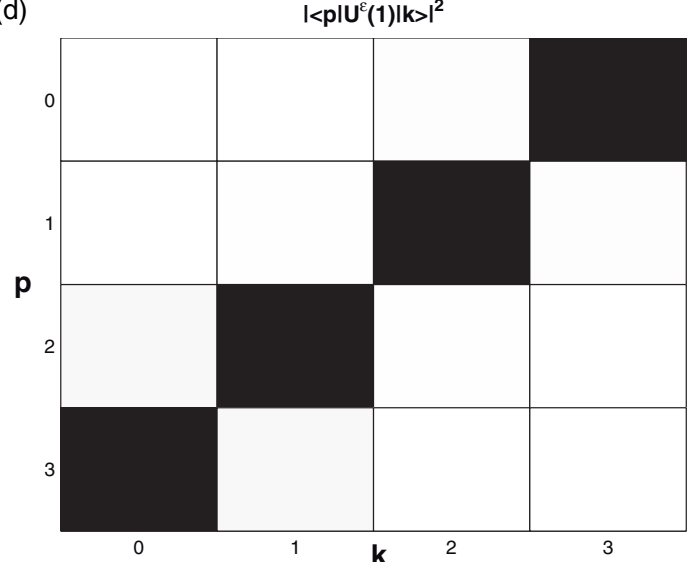

Figure 1. Control scheme transferring $|k\rangle$ to $|N-k-1\rangle$. (a) Control inputs $A(s), \omega(s)$. (b) $s$-dependent eigenvalues of $H(s)$ (thick lines) and of $H_{R}(\omega(s))$ (thin lines). (c) Population on level $|3\rangle$ for ten systems whose parameters $\mu_{0}, \mu_{1}, \mu_{2}$ and $\Delta_{1}, \Delta_{2}, \Delta_{3}$ were randomly picked respectively in $[1,5]$ and $[-0.4,0.4]$, and all starting at the initial state $|0\rangle$. (d) Squared norm of the matrix elements of $U^{\varepsilon}(1)$, represented in shading from white (value 0 ) to black (value 1 ).

\subsection{From $|0\rangle$ to any $|p\rangle$}

For the sake of clarity, we start by giving sufficient conditions on $A$ and $\omega$ for the particular population transfer from $|0\rangle$ to arbitrary level $|p\rangle$. Section 4.2 generalizes the result to an arbitrary initial state $|l\rangle$. Consider the following assumptions:

(A1) $\mathcal{S}$ is an ensemble of systems of type (10) with $\mu_{j} \in$ $\left[\mu_{\min }, \mu_{\max }\right]$ for all $j \in \mathbb{N}_{0}^{N-2}$, for some given $\mu_{\max }>$ $\mu_{\min }>0$, and with given sequence of detunings $\left(\Delta_{0}, \ldots, \Delta_{N-1}\right)$, such that the set $\left\{k\left(\Delta_{k}-v\right): k \in \mathbb{N}_{0}^{N-1}\right\}$ contains at least $N-1$ distinct values for any $v \in \mathbb{R}$;

(A2) $\omega$ is analytic and $\frac{\mathrm{d}}{\mathrm{d} s} \omega(s)>\gamma>0$ for all $s \in[0,1]$;

(A3) $\omega(0)$ and $\omega(1)$ are such that (13) holds.

For any $m$ and $n$ in $\mathbb{N}_{0}^{N-1}$ with $m<n$, we denote by $s(m, n)$ the unique time ${ }^{5}$ where $\lambda_{m}^{R}(s(m, n))=\lambda_{n}^{R}(s(m, n))$.

As all systems in $\mathcal{S}$ have the same sequence of detunings, they feature the same eigenvalues $\lambda_{0}^{R}, \ldots, \lambda_{N-1}^{R}$ of $H_{R}$ and hence the same set of $s(m, n)$. The set of all $s(m, n)$ equals $\mathcal{I}^{\omega}$ defined in (14), with dependence on a particular system $\in \mathcal{S}$ becoming irrelevant. The end of (A1) further implies that $H_{R}$ has at most one pair of equal eigenvalues for any $s \in[0,1]$, i.e. $(m, n) \neq(j, k)$ implies $s(m, n) \neq s(j, k)$; hence, $\mathcal{I}^{\omega}$ contains $N(N-1) / 2$ distinct values. Further

5 If assumptions (A1) to (A3) hold, then the existence and unicity of $s(m, n)$ is ensured for all $m$ and $n>m$ : see figure 2(b) or figure 3(c). define $\mathcal{I}_{0}^{\omega}=\left\{s_{1}, \ldots, s_{N-1}\right\} \subset \mathcal{I}^{\omega}$ the $N-1$ points where $\lambda_{0}^{R}(s)=\lambda_{n}^{R}(s)$ for some $n \in \mathbb{N}_{1}^{N-1}$, numbered such that $s_{1}<s_{2}<\cdots<s_{N-1}$. Thus, for each $s_{k} \in \mathcal{I}_{0}^{\omega}$ there exists a unique $n \in \mathbb{N}_{1}^{N-1}$ such that $s_{k}=s(0, n)$.

The key requirements on the control to achieve population transfer from $|0\rangle$ to $|p\rangle$ are (i) to use a sufficiently chirped pulse frequency-condition (A3) - and (ii) to shape the pulse amplitude in order to appropriately provoke-(c) in theorem 2-or avoid-(b), (d) in theorem 2-crossing of eigenvalues of $H$.

Theorem 2. Consider $\mathcal{S}$ an ensemble of systems satisfying (A1) with a control $\omega$ satisfying (A2) and (A3). Take $p \in$ $\{0, \ldots, N-1\}$ and consider a control $A$ with the following properties.

(a) $A$ is analytic over $[0,1]$ and $A(0)=A(1)=0$.

(b) $A(s) \neq 0$ for all $s \in \mathcal{I}^{\omega} \backslash \mathcal{I}_{0}^{\omega}$.

(c) $A\left(s_{k}\right)=0$ for all $s_{k} \in \mathcal{I}_{0}^{\omega}$ with $k \leqslant N-p-1$.

(d) $A\left(s_{k}\right) \neq 0$ for all $s_{k} \in \mathcal{I}_{0}^{\omega}$ with $k \geqslant N-p$.

Then $\exists$ a constant $C>0$ such that, for all $\varepsilon>0$,

$$
\sup _{\mathcal{S}} \| U^{\varepsilon}(1)|0\rangle\left\langle 0\left|U^{\varepsilon}(1)^{\dagger}-\right| p\right\rangle\langle p| \| \leqslant C \sqrt{\varepsilon} .
$$

The proof, given in section 6, shows that at eigenvalue crossing points the system adiabatically follows the eigenvector corresponding to the crossing branch. 


\subsection{From any $|l\rangle$ to any $|p\rangle$}

Under assumptions (A1)-(A3), we denote $\mathcal{I}_{k+}^{\omega}(s)=$ $\left\{s(m, n) \in \mathcal{I}^{\omega}: m=k, n>k\right.$ and $\left.s(m, n)>s\right\}$ and $\mathcal{I}_{k-}^{\omega}(s)=\left\{s(m, n) \in \mathcal{I}^{\omega}: m<k, n=k\right.$ and $\left.s(m, n)>s\right\}$, for any $k \in \mathbb{N}_{0}^{N-1}$. Further let $q_{k \pm}(s)=\inf \left(\mathcal{I}_{k \pm}^{\omega}(s)\right)$ and define $g_{k \pm}(s)$ by $s\left(k, g_{k+}(s)\right)=q_{k+}(s)$ and $s\left(g_{k-}(s), k\right)=q_{k-}(s)$ respectively. For $p \leqslant N-l-1$, construct $\mathcal{I}_{l p}^{\omega}$ with the following algorithm:

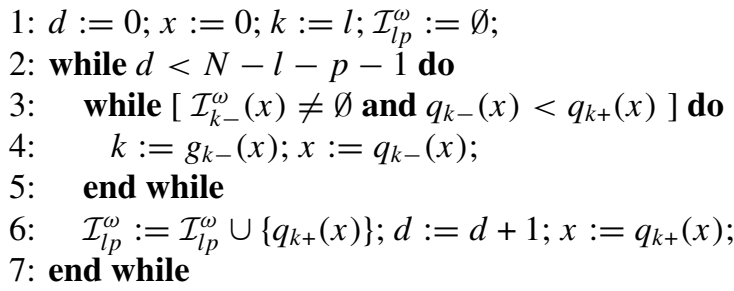

The algorithm always successfully completes ${ }^{6}$. For $p \geqslant N-l-1$, we can define $\mathcal{I}_{l p}^{\omega}$ with a similar algorithm but where ' $<$ ' is changed to ' $>$ ' on line 2 and indices $k_{-}, k_{+}$are switched. Then $\mathcal{I}_{l p}^{\omega}$ contains $|N-l-p-1|$ elements.

Corollary 1. Consider $\mathcal{S}$ an ensemble of systems satisfying (A1) with a control $\omega$ satisfying (A2) and (A3). Take l, $p$ in $\mathbb{N}_{0}^{N-1}$ and consider a control $A$ with the following properties:

(a) $A$ is analytic over $[0,1]$ and $A(0)=A(1)=0$.

(b) $A(s)=0$ for all $s \in \mathcal{I}_{l p}^{\omega}$.

(c) $A(s) \neq 0$ for all $s \in \mathcal{I}^{\omega} \backslash \mathcal{I}_{l p}^{\omega}$.

Then $\exists$ a constant $C>0$ such that, for all $\varepsilon>0$,

$$
\sup _{\mathcal{S}} \| U^{\varepsilon}(1)|l\rangle\left\langle l\left|U^{\varepsilon}(1)^{\dagger}-\right| p\right\rangle\langle p| \| \leqslant C \sqrt{\varepsilon} .
$$

Assumption (A1) ensures that each eigenvalue crossing/anti-crossing can be addressed individually. This ensures that any transfer can be implemented in any situation, but it is in general not necessary for a given system and transfer, as (simultaneous) crossings of some eigenvalue branches are irrelevant. The control proposed for theorem 2 or corollary 1 is just one amongst many possibilities of 'eigenvalue crossing designs'. Indeed, depending on $(l, p)$ and on the particular arrangement of $s(m, n)$, one can find other subsets $\mathcal{J}_{l p} \subset \mathcal{I}^{\omega}$ such that taking $A(s)=0$ if and only if $s \in \mathcal{J}_{l p}$ permutes the eigenvalues in such a way that $\lambda_{l}(1)=\lambda_{p}^{R}(1)$ (see section 6 for the formal definition of $\left.\lambda_{l}(1)\right)$. The controls that we propose are optimal in the sense that they require a minimal number of pairwise crossings, that is, of annihilations of $A$ at accurate points. Variant annihilation subsets $\mathcal{J}_{l p}$ may be useful (i) to avoid some crossing points $s(m, n)$ or eigenvalue branches (e.g. because corresponding $\Delta_{m}$ or $\Delta_{n}$ is poorly known, or because $s(m, n)$ is close to some other point in $\left.\mathcal{I}^{\omega}\right)$, (ii) to optimize adiabatic convergence as a function of $\varepsilon$ or (iii) to simultaneously perform population transfers between several eigenstates, as we do in section 5.

Another approach [27] for transferring $|l\rangle$ to $|p\rangle$ is to use $A(s)$ Gaussian, i.e. without any annihilations, but

\footnotetext{
6 Indeed by construction, the cardinality of $\mathcal{I}_{k+}^{\omega}(x)$ equals $N-l-d-1$ (except during the update on line 6) and the cardinality of $\mathcal{I}_{k-}^{\omega}(x)$ decreases by one each time line 4 is applied; thus it is impossible to keep applying line 4 infinitely, and line 6 is always well defined (that is $\mathcal{I}_{k+}^{\omega}(x) \neq \emptyset$ ) for $d<N-l-1$.
}

reduce $\omega(s)$ to a specific range. Indeed, under the above assumptions, it is possible to choose $\omega_{\min }$ and $\omega_{\max }$ such that $l\left(\Delta_{l}-\bar{v}\right)=p\left(\Delta_{p}-\bar{v}\right)$ for some $\bar{v} \in\left[\omega_{\min }, \omega_{\max }\right]$ and $H_{R}(v)$ is non-degenerate for all $v \in\left[\omega_{\min }, \omega_{\max }\right] \backslash\{\bar{v}\}$. Then taking $\omega(s)$ monotone between $\omega_{\min }$ and $\omega_{\max }$ just induces one avoided crossing that exchanges $|l\rangle$ and $|p\rangle$. Pictorially, this is like selecting a particular narrow vertical slice in figure 1(b). Depending on the specific system under study and whether it is experimentally easier to precisely modulate the amplitude or the phase of a field, one method may be more suitable than the other. A main advantage of our method is that, unlike the method proposed in [27], it can be extended to achieve any permutation of eigenstates as is shown in section 5 .

\subsection{Simulations}

As in section 3.2 we simulate (10) for a four-level quantum ladder (so $N=4$ ) with $\mu_{0}, \mu_{1}, \mu_{2} \in\left[\mu_{\min }, \mu_{\max }\right]=[1,5]$. We now take $\varepsilon=10^{-3}$ and in accordance with the statement of theorem 2 we fix the anharmonicities, taking $\Delta_{1}=-1$, $\Delta_{2}=0.3, \Delta_{3}=0$ (the value of $\Delta_{0}$, multiplied by $k=0$, is irrelevant). We target in particular a transfer from $|0\rangle$ to $|2\rangle$. The algorithm of section 4.2 reduces to the simple case of theorem 2, requesting a single zero of $A(s)$ at $s=\inf \{s(0,1), s(0,2), s(0,3)\}=s(0,1)=0.25$ in addition to $A(0)=A(1)=0$. We take $A(s)=s(1-s)(s-0.25)$ and $\omega(s)=4\left(s-\frac{1}{2}\right)$, represented in figure 2(a). Figure 2(b) shows how the eigenvalues $\lambda_{k}(s)$ of $H(s)$ cross or not (thick lines); the eigenvalues of $H_{R}(\omega(s))$ (thin lines) define points $s(m, n)$ for our control design. Figure 2(d) confirms achievement of the intended result by showing the squared norm of components of the matrix $U^{\varepsilon}(1)$ in the basis $(|0\rangle, \ldots,|3\rangle)$ : we indeed have $\left|\left\langle p\left|U^{\varepsilon}(1)\right| k\right\rangle\right|^{2} \approx 1$ for $(p, k)=(2,0)$ (other values incidental). Figure 2(c) illustrates ensemble control on ten systems with different random values of $\mu_{0}, \mu_{1}, \mu_{2}$. Since for this particular case the control only exploits precise crossing point $s(0,1)=0.25$, we might actually allow ensembles with different $\Delta_{2}, \Delta_{3}$.

\section{Robust ensemble permutation of populations}

In this section we describe the most general result of the paper, adiabatically transferring $(|0\rangle\langle 0|, \ldots| N-1\rangle,\langle N-1|)$ to $(|\sigma(0)\rangle\langle\sigma(0)|, \ldots,| \sigma(N-1)\rangle\langle\sigma(N-1)|)$, where $\sigma$ is any permutation of $\mathbb{N}_{0}^{N-1}$. As in section 4 , the population permutation works on an ensemble of systems with different values of $\mu_{0}, \ldots, \mu_{N-2}$ (dipole moments), and for a general class of inputs where zero-crossings of $A(s)$ must be correlated with degeneracies of $H_{R}(\omega(s))$; the latter depend on $\omega(s)$ and require anharmonicities $\Delta_{0}, \ldots, \Delta_{N-1}$ to be fixed and known. We prove existence of an appropriate control by recurrence on $N$. In fact this recurrence method can be used to design $A(s)$, as we illustrate in section 5.2.

\subsection{Permutation theorem}

Theorem 3. Consider $\mathcal{S}$ an ensemble of systems satisfying (A1) with a control $\omega$ satisfying (A2) and (A3). Take $\sigma$ any 
(a)

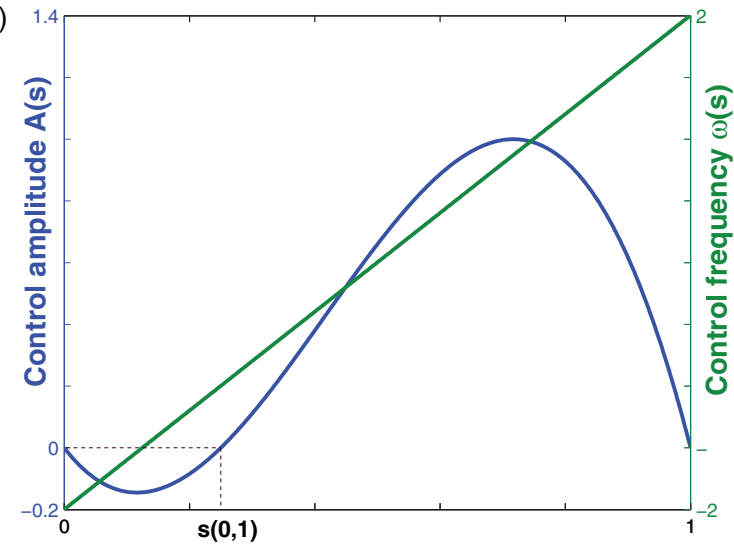

(c)

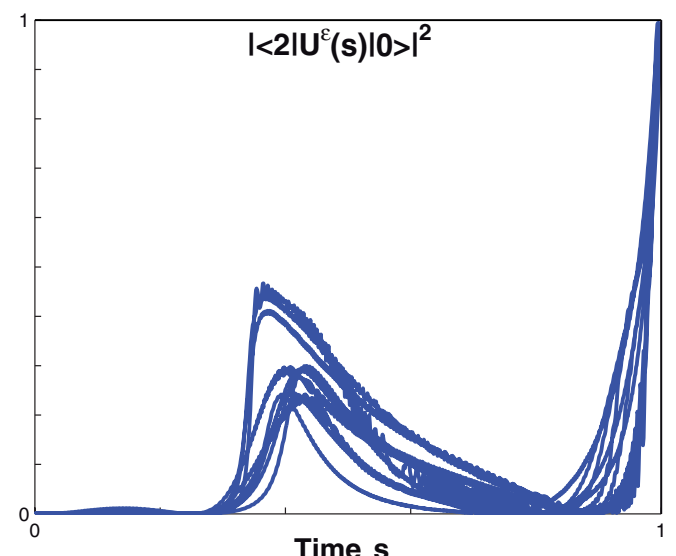

(b)

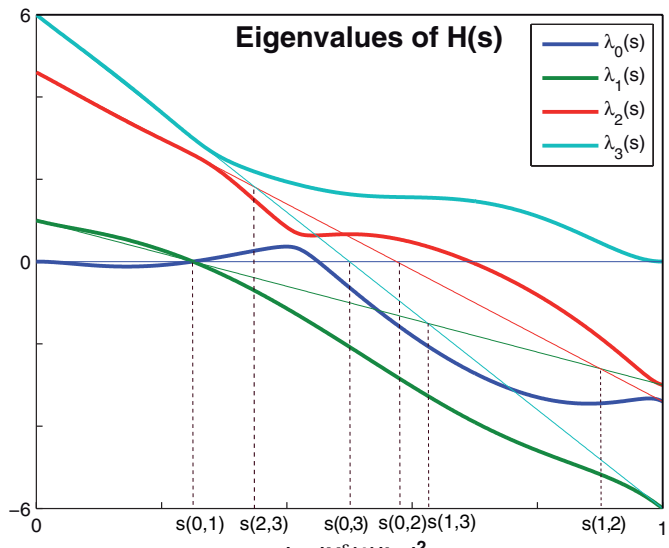

(d)

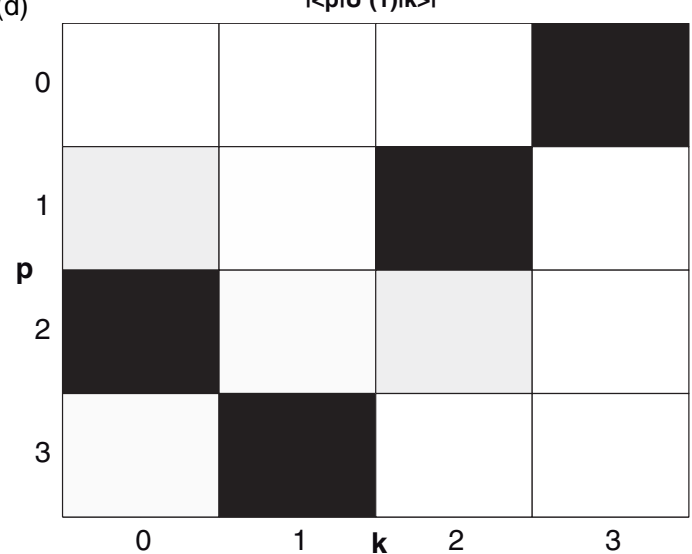

Figure 2. Control scheme transferring $|0\rangle$ to $|2\rangle$; subplots analogous to figure 1, except that $\Delta_{1}, \Delta_{2}, \Delta_{3}$ remain fixed for (c). $A(s)$ vanishes at $s=0.25$ so that $\lambda_{0}$ and $\lambda_{1}$ cross instead of avoiding crossing.

permutation of $\mathbb{N}_{0}^{N-1}$. Then there exists a subset $\mathcal{I}_{A} \subseteq \mathcal{I}^{\omega}$ for which, taking control A to satisfy

(a) A analytic over $[0,1]$ and $A(0)=A(1)=0$,

(b) $A(s)=0$ for all $s \in \mathcal{I}_{A}$,

(c) $A(s) \neq 0$ for all $s \in \mathcal{I}^{\omega} \backslash \mathcal{I}_{A}$,

implies that $\exists$ a constant $C>0$ such that, for all $\varepsilon>0$,

$$
\sup _{\substack{\mathcal{S} \\ k \in \mathbb{N}_{0}^{N-1}}} \| U^{\varepsilon}(1)|k\rangle\left\langle k\left|U^{\varepsilon}(1)^{\dagger}-\right| \sigma(k)\right\rangle\langle\sigma(k)| \| \leqslant C \sqrt{\varepsilon} .
$$

Since the proof of this theorem is constructive and necessary for the understanding of the example below, we present it here.

Proof. (of theorem 3). The formal arguments (sup, adiabatic propagator) are presented in detail in the proof of theorem 1 in section 6. We focus on the construction of the control $A(s)$ by following analytic eigenvalue branches $\lambda_{k}(s)$ of $H(s)$.

The property is obvious for $N=2$ : either $(\sigma(0), \sigma(1))=$ $(1,0)$, which follows theorem 1 just requiring $A(s(0,1)) \neq 0$; or $(\sigma(0), \sigma(1))=(0,1)$, which follows theorem 2 transferring $|0\rangle$ to $|p\rangle=|0\rangle$ with one crossing ${ }^{7}$, i.e. just requiring $A(s(0,1))=0$.

7 Indeed, $\left\{P_{\lambda_{0}(1)}, P_{\lambda_{1}(1)}\right\}=\{|0\rangle\langle 0|| 1\rangle,\langle 1|\}$ then automatically implies transferring $|1\rangle\langle 1|$ to $P_{\lambda_{1}(1)}=|1\rangle\langle 1|$.
Assume that we can achieve any permutation of $\mathbb{N}_{0}^{K-1}$ for $N=K$, and we are given a permutation $\sigma$ of $\mathbb{N}_{0}^{K}$ for $N=K+1$ where $\sigma(l)=K$ and $\sigma(K)=p$.

- If $l=p=K$, i.e. $\sigma(K)=K$, then first build the remaining permutation on levels $|0\rangle, \ldots,|K-1\rangle$ by neglecting level $|K\rangle$. This uses the result for $N=K$; it just requires $A(s)=0$ for some $s=s(m, n)$ and $A(s) \neq 0$ for some other $s=s(m, n)$, with $m, n<K$. Now take a particular such $A(s)$ where in addition, $A(s)=0$ for all $s \in\left\{s(m, K): m \in \mathbb{N}_{0}^{K-1}\right\}$. Then $\lambda_{K}(s)$, starting at $\lambda_{K}(0)=\lambda_{K}^{R}(0)$, exactly follows the same crossings as $\lambda_{K}^{R}(s)$ to end up as $\lambda_{K}(1)=\lambda_{K}^{R}(1)$; the other levels remain unperturbed, so $\sigma$ is achieved.

- If $l \neq K \neq p$, then first construct $\bar{A}(s)$ by applying the result of the preceding point to $\bar{\sigma}$, defined by

$\bar{\sigma}(l)=p ; \quad \bar{\sigma}(K)=K ; \quad \bar{\sigma}(k)=\sigma(k)$ for all $k \notin\{l, K\}$.

$\bar{A}(s)$ performs the target permutation, except that $K$ remains on $K$ and $l$ goes to $p$. From (13) the eigenvalue branch $\lambda_{K}(s)$ necessarily crosses, at some $\bar{s} \in\{s(m, K)$ : $\left.m \in \mathbb{N}_{0}^{K-1}\right\}$, the analytic eigenvalue branch that starts at $\lambda_{l}(0)=\lambda_{l}^{R}(0)$ and ends at $\lambda_{l}(1)=\lambda_{p}^{R}(0)$. Define $A(s)$ to have the same zeros as $\bar{A}(s)$ except that $A(\bar{s}) \neq 0$. This just transforms the crossing at $\bar{s}$ into an anti-crossing, such that the analytic branch coming from $\lambda_{K}(0)$ (respectively 
(a)

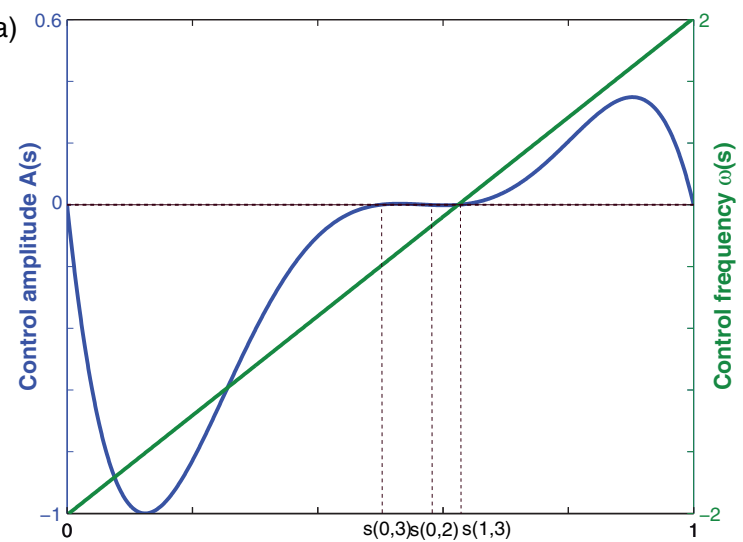

(c)

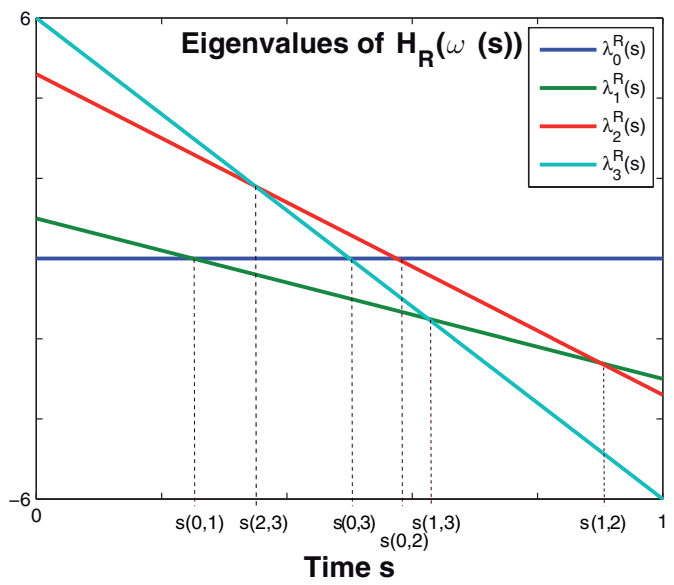

(b)

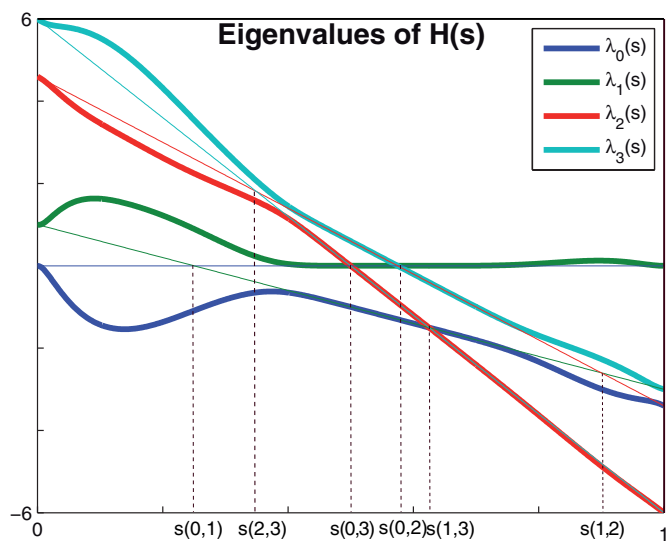

(d)

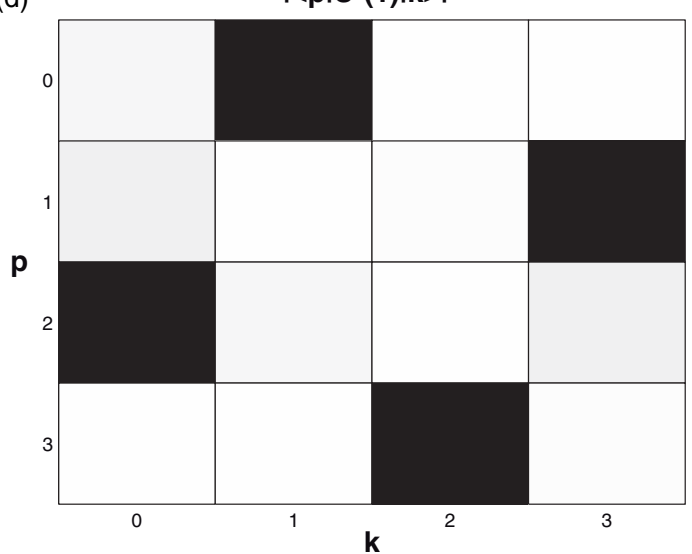

Figure 3. Control scheme generating permutation $\sigma(0,1,2,3)=(2,0,3,1)$ and simulation result. Subplots (a),(b),(d) are analogous to figure 1. Subplot (c) shows the eigenvalues of $H_{R}(\omega(s))$, used to design $\mathcal{I}_{A}$ (see text). The points $s \in \mathcal{I}_{A}$ where $A(s)=0$ are marked on (a).

$\left.\lambda_{l}(0)\right)$ now connects to the analytic branch going to $\lambda_{p}(1)$ (respectively $\lambda_{K}(1)$ ). Thus $A(s)$ achieves the target permutation $\sigma$.

Each 'eigenvalue crossing design' choice $\mathcal{I}_{A}$ yields a particular permutation $\sigma_{\mathcal{I}_{A}}$. For $N>2$, the number $2^{N(N-1) / 2}$ of possible $\mathcal{I}_{\mathcal{A}}$ (i.e. subsets of $\mathcal{I}^{\omega}$ ) is strictly larger than the number $N$ ! of permutations. Thus there are still several $\mathcal{I}_{\mathcal{A}}$ that yield the same $\sigma$. Unlike in section 4 , building $A(s)$ as in the proof of theorem 3 does not necessarily yield a minimal cardinality of $\mathcal{I}_{\mathcal{A}}$ for given $\sigma$.

\subsection{Example and simulations}

We first illustrate the control design by recurrence based on the proof of theorem 3 . Consider the target permutation $\sigma(0,1,2,3)=(2,0,3,1)$. First we reduce it down to an elementary permutation. Start with $K=N-1=3$ and note $(l, p)=(2,1)$ because $\sigma(2)=K$ and $\sigma(K)=1$; we thus define $\bar{\sigma}(0,1, l=2,3)=(2,0, p=1,3)$ and impose $\bar{A}(s)=0$ for $s \in\{s(0,3), s(1,3), s(2,3)\}$ reducing the permutation to $0,1,2$. Then we take $\bar{K}=N-1=2$ and note $(\bar{l}, \bar{p})=(0,1)$ because $\bar{\sigma}(0)=\bar{K}$ and $\bar{\sigma}(\bar{K})=1$; we thus define $\overline{\bar{\sigma}}(\bar{l}=0,1,2,3)=\overline{\bar{\sigma}}(\bar{p}=1,0,2,3)$ and impose $\overline{\bar{A}}(s)=0$ for $s \in\{s(0,2), s(1,2)\}$ reducing the permutation to 0,1 . To implement $\overline{\bar{\sigma}}$ we need $\overline{\bar{A}}(s(0,1)) \neq 0$. Now we progressively move up to permutations on more levels, removing one ${ }^{-}$at a time from our objects. The reader is encouraged to follow crossings/anti-crossings under the different controls by referring to figure 3(c), corresponding to our chirping choice $\omega(s)=4\left(s-\frac{1}{2}\right)$. Under $\overline{\bar{A}}$ the analytic branch from $|\bar{l}\rangle=|0\rangle$ to $|\bar{p}\rangle=|1\rangle$ meets the branch staying on $|\bar{K}\rangle=|2\rangle$ at $\overline{\bar{s}}=s(1,2)$. We therefore impose $\bar{A}(s(1,2)) \neq 0$ unlike for $\overline{\bar{A}}$, and for the rest copy the requirements of $\overline{\bar{A}}$ : $\bar{A}(s(0,1)) \neq 0, \bar{A}(s(0,2))=0$. Now under $\bar{A}$ the branch from $|l\rangle=|2\rangle$ to $|p\rangle=|1\rangle$ crosses the branch staying on $|K\rangle=|3\rangle$ at $\bar{s}=s(2,3)$. We therefore get requirements for our actual control $A$ by imposing $A(s(2,3)) \neq 0$ unlike for $\bar{A}$, for the rest copying the requirements of $\bar{A}$, i.e. $A(s)=0$ for $s \in\{s(0,3), s(1,3), s(0,2)\}$ and $A(s) \neq 0$ for $s \in\{s(0,1), s(1,2)\}$. To satisfy these requirements, we take the polynomial control $A(s)=s(1-s)(s-s(0,3))(s-$ $s(1,3))(s-s(0,2))$, represented in figure 3(a). Figure 3(b) shows how the eigenvalues of $H(s)$ cross and anti-cross depending on whether $A(s)$ vanishes or not. The squared norm components of $U^{\varepsilon}(1)$ resulting from a simulation of (10) with this control and $\varepsilon=10^{-3}$ are shown in figure 3(d) on a white-to-black scale, confirming achievement of permutation $\sigma(0,1,2,3)=(2,0,3,1)$.

Figure 4 shows the same squared norm components of $U^{\varepsilon}(1)$ in grey shades for 24 cases, corresponding to different 

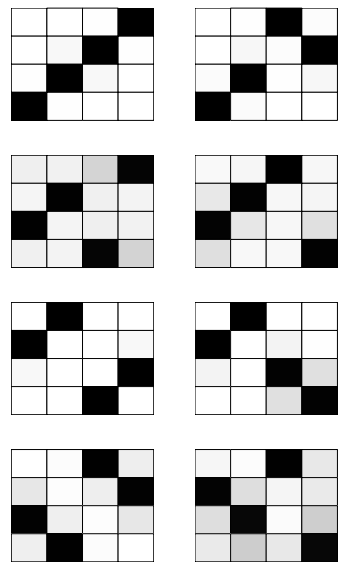
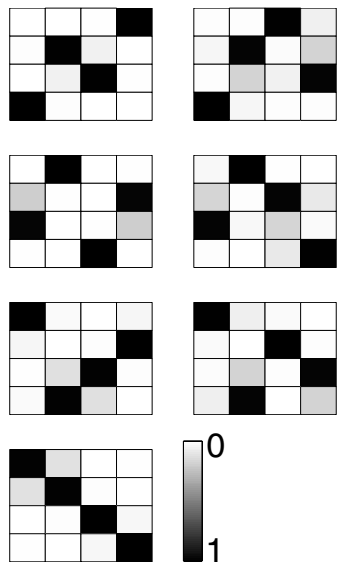
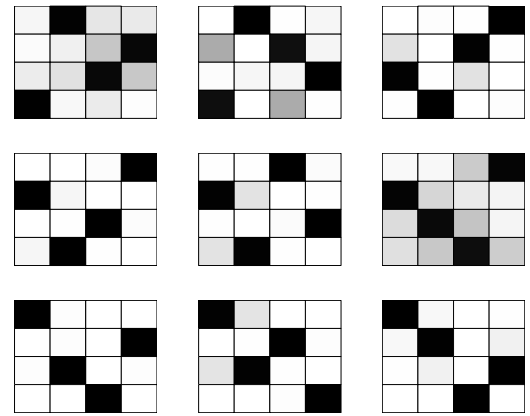

Figure 4. Simulation results of (10) for 24 different controls $A(s)$ following theorem 3 to achieve each one of the 24 permutations of $(0,1,2,3)$ with adiabatic passage. Shading represents squared norm of elements of matrix $U^{\varepsilon}(1)$ expressed in the basis $|0\rangle, \ldots,|3\rangle$, from white (value 0) to black (value 1). In other words, each subplot may be read as a $4 \times 4$ matrix where the black patches are ones and the white patches are zeros; gray patches indicate intermediate values, reflecting that the unitary propagator obtained by integrating (10) is not exactly a permutation matrix for the finite $\varepsilon=10^{-3}$, the permutation becoming perfect for infinitesimal $\varepsilon$ according to our theorems.

control inputs $A(s)$ designed for all 24 possible permutations of the set $(0,1,2,3)$. The controls $A(s)$ are built as the product of (i) a polynomial vanishing on $\mathcal{I}_{A} \cup\{0,1\}$ and only there, and (ii) a set of functions $(1+g(s-s(m, n)))$, with $g(s-s(m, n))$ Gaussians centred on all $s \in \mathcal{I}_{\omega} \backslash \mathcal{I}_{A}$; the role of the latter is to amplify $A(s)$ in the vicinity of intended 'anti-crossings', improving convergence of the adiabatic limit as a function of $\varepsilon$. Figure 4 corresponds to the choice $\varepsilon=10^{-3}$.

\section{Proofs}

In this section we give the proofs of all the formal results presented in previous sections.

\subsection{Proof of theorem 1}

We start the proof by recalling the following result [28].

Lemma 1. Let $D_{N}$ be a real tridiagonal and symmetric $N \times N$ matrix defined by

$$
D_{N}=\sum_{k=0}^{N-1} a_{k}|k\rangle\langle k|+\sum_{k=0}^{N-2} c_{k}(|k\rangle\langle k+1|+| k+1\rangle\langle k|)
$$

in some orthonormal basis $(|0\rangle, \ldots,|N-1\rangle)$. If $c_{k} \neq 0$ for all $k \in \mathbb{N}_{0}^{N-2}$, then $D_{N}$ is non-degenerate.

Proof (of lemma 1). Denote by $Q_{n}$ the characteristic polynomial of $D_{n}$, which is defined as (15) with $N$ replaced by $n$, for all $n \in\{1, \ldots, N\}$. The sequence of polynomials $\left(Q_{n}\right)_{n}$ verifies the following recurrence relation: for $n \geqslant 2$,

$$
Q_{n}(x)=\left(x-a_{n-1}\right) Q_{n-1}(x)-\left(c_{n-2}\right)^{2} Q_{n-2}(x),
$$

with $Q_{0}(x)=1$ and $Q_{1}(x)=x-a_{0}$. According to Favard's theorem [22], a sequence verifying (16) where $\left(c_{n-2}\right)^{2}>0$ for all $n$ is a sequence of orthogonal polynomials. Furthermore, from [23], theorem 3.3.1, every polynomial $Q_{n}$ in a sequence of orthogonal polynomials has $n$ real and distinct zeros; this is in particular true for $n=N$; therefore, $D_{N}$ is non-degenerate.
Proof (of theorem 1). We prove the result for any single system in $\mathcal{S}$ and conclude that it remains true for the sup over $\mathcal{S}$. Indeed, the application

$$
\begin{aligned}
& \left(\mu_{0}, \ldots, \mu_{N-2}, \Delta_{0}, \ldots, \Delta_{N-1}\right) \\
& \quad \rightarrow \quad \| U^{\varepsilon}(1)|k\rangle\left\langle k\left|U^{\varepsilon}(1)^{\dagger}-\right| N-k-1\right\rangle\langle N-k-1| \|
\end{aligned}
$$

reaches its sup over the allowed compact space since the state of a (sufficiently regular) dynamical system at a finite time depends continuously on system parameters (see e.g. [29], theorem 3.5). The proof for one system is in two steps: first we prove that the hypotheses of the adiabatic theorem with gap condition are verified; then we apply the theorem to compute the image at $s=1$ of an initial projector $|k\rangle\langle k|$ in adiabatic approximation.

Step 1: by hypothesis (a), we have $H(s) \in \mathcal{C}^{2}\left([0,1], \mathcal{H}_{N}\right)$ and therefore continuous over [0,1]. From [30], section II.5.2, it is then possible to find $N$ continuous functions $\lambda_{0}(s), \ldots, \lambda_{N-1}(s)$ such that $\lambda_{0}(s) \leqslant \ldots \leqslant \lambda_{N-1}(s)$ for all $s \in[0,1]$ are the eigenvalues of $H(s)$. In terms of associated eigenspace projections, note that $\left\{P_{\lambda_{k}(s)}: k \in \mathbb{N}_{0}^{N-1}\right\}=\left\{|k\rangle\langle k|: k \in \mathbb{N}_{0}^{N-1}\right\}$ every time $A(s)=0$ and $H(s)$ is non-degenerate, by unicity of the spectral decomposition of a non-degenerate matrix. However, the pairwise correspondence depends on the value of $\omega(s)$. In particular, by hypotheses (b) and (c),

$P_{\lambda_{k}(0)}=|k\rangle\langle k|$ and $P_{\lambda_{k}(1)}=|N-k-1\rangle\langle N-k-1|$

for all $k$. For a given $s \in[0,1]$,

- either $A(s) \neq 0$, then $H(s)$ has $N$ distinct eigenvalues according to lemma 1 ;

- or $A(s)=0$, then $H(s)=H_{R}(\omega(s))$ and it must have $N$ distinct eigenvalues by hypothesis $\left(\mathrm{c}^{\prime}\right)$. Hence,

$$
\lambda_{0}(s)<\cdots<\lambda_{N-1}(s) \text { for all } s \in[0,1] .
$$


Then by continuity over the compact $[0,1]$, there exists $\delta>0$ such that $\lambda_{k}(s)+\delta<\lambda_{k+1}(s)$ for all $k \in \mathbb{N}_{0}^{N-2}$ and for all $s \in[0,1]$ : each $\lambda_{k}(s)$ is at all times surrounded by a 'spectral gap' of amplitude $\delta$ in which there is no other eigenvalue. We can therefore apply the adiabatic theorem with gap condition (see [13], theorem 2.2) to eigenvalue $\lambda_{k}(s)$, for any particular $k \in \mathbb{N}_{0}^{N-1}$, as is done in the following.

Step 2: the adiabatic theorem ensures that $P_{\lambda_{k}(s)} \in$ $\mathcal{C}^{2}\left([0,1], \mathcal{H}_{N}\right)$. Define the 'adiabatic Hamiltonian'

$H_{a, k}(s)=H(s)-\mathrm{i} \varepsilon P_{\lambda_{k}(s)} \frac{\mathrm{d}}{\mathrm{d} s} P_{\lambda_{k}(s)}-\mathrm{i} \varepsilon P_{\lambda_{k}(s)}^{\perp} \frac{\mathrm{d}}{\mathrm{d} s} P_{\lambda_{k}(s)}^{\perp}$

where $P_{\lambda_{k}(s)}^{\perp}=I-P_{\lambda_{k}(s)}$, and the 'adiabatic propagator' $U_{a, k}^{\varepsilon}$ which verifies, for all $s \in[0,1]$,

i $\varepsilon \frac{\mathrm{d}}{\mathrm{d} s} U_{a, k}^{\varepsilon}(s)=H_{a, k}(s) U_{a, k}^{\varepsilon}(s) \quad$ with $U_{a, k}^{\varepsilon}(0)=I$.

One verifies that this construction ensures

$$
U_{a, k}^{\varepsilon}(s) P_{\lambda_{k}(0)} U_{a, k}^{\varepsilon}(s)^{\dagger}=P_{\lambda_{k}(s)}
$$

for all $s \in[0,1]$. The adiabatic theorem states the existence of a constant $C_{1}>0$ such that

$$
\left\|U^{\varepsilon}(s)-U_{a, k}^{\varepsilon}(s)\right\| \leqslant C_{1} \varepsilon \text { for all } s \in[0,1],
$$

in particular for $s=1$. This implies

$$
\begin{aligned}
& \| U^{\varepsilon}(1)|k\rangle\left\langle k\left|U^{\varepsilon}(1)^{\dagger}-U_{a, k}^{\varepsilon}(1)\right| k\right\rangle\langle k| U_{a, k}^{\varepsilon}(1)^{\dagger} \| \\
& \leqslant \|\left(U^{\varepsilon}(1)-U_{a, k}^{\varepsilon}(1)\right)|k\rangle\langle k| U^{\varepsilon}(1)^{\dagger} \| \\
& +\| U_{a, k}^{\varepsilon}(1)|k\rangle\langle k|\left(U^{\varepsilon}(1)-U_{a, k}^{\varepsilon}(1)\right)^{\dagger} \| \\
& \leqslant\left\|U^{\varepsilon}(1)-U_{a, k}^{\varepsilon}(1)\right\| \||k\rangle\langle k| \|\left(\left\|U_{a, k}^{\varepsilon}(1)\right\|+\left\|U^{\varepsilon}(1)\right\|\right) \\
& \leqslant C_{1} \varepsilon \cdot 1 \cdot 2 \sqrt{N}
\end{aligned}
$$

since $\|U\|=\sqrt{\operatorname{tr} U^{\dagger} U}=\sqrt{\operatorname{tr} I}$ for any unitary matrix $U$. Combining this with (17), (21) yields the result, where $C=2 C_{1} \sqrt{N}$.

\subsection{Proof of theorem 2 and corollary 1}

We start by proving a lemma about the behaviour of timedependent eigenvalues crossing each other.

Lemma 2. Assume that $H(s)$ as defined in (11) depends analytically on the real parameter $s$ on an interval $\mathcal{I} \subset \mathbb{R}$, with $\frac{\mathrm{d}}{\mathrm{d} s} \omega(s)>\gamma>0$ for all $s \in \mathcal{I}$. Suppose that $H_{R}(\omega(s))$ is non-degenerate on $\mathcal{I}$ except for a simple degeneracy at $\bar{s} \in \mathcal{I}$, i.e. $H_{R}(\omega(\bar{s}))$ has $N-1$ distinct eigenvalues and $H_{R}(\omega(s))$ has $N$ distinct eigenvalues for $s \in \mathcal{I} \backslash\{\bar{s}\}$. If $A(\bar{s})=0$, then:

(a) there exist $N$ unique functions $\lambda_{0}, \ldots, \lambda_{N-1}$ analytic over $\mathcal{I}$, with $\lambda_{0}(s)<\cdots<\lambda_{N-1}(s)$ for all $s<\bar{s}$, and such that $\left\{\lambda_{0}(s), \ldots, \lambda_{N-1}(s)\right\}$ are the eigenvalues of $H(s)$ for all $s \in \mathcal{I}$.

(b) Let $k$ be such that $\lambda_{k}(\bar{s})=\lambda_{k+1}(\bar{s})$. Then for all $s>\bar{s}$ we have

$$
\lambda_{0}(s)<\cdots<\lambda_{k+1}(s)<\lambda_{k}(s)<\cdots<\lambda_{N-1}(s) .
$$

Proof (of lemma 2). Point (a) is a direct consequence of [30], theorem 6.1. The order of the analytic eigenvalues is obviously preserved over time intervals where $H(s)$ is non-degenerate; by lemma 1 , these intervals are $\{s<\bar{s}\}$ and $\{s>\bar{s}\}$. The issue is what happens at $s=\bar{s}$. In the following, we show that $\lambda_{k}^{\prime}(\bar{s}) \neq \lambda_{k+1}^{\prime}(\bar{s})$. Since the eigenvalues are analytic and $\lambda_{k}(\bar{s})=\lambda_{k+1}(\bar{s})$, a Taylor expansion then yields the conclusion of (b).

We lead calculations similar to those of [14], section XVI.II.8. According to [30], section II.6.2, since $H$ is analytic over $\mathcal{I}$ and $H(s) \in \mathcal{H}_{N}$ for all $s \in \mathcal{I}$, there exist rank-one orthogonal spectral projections $P_{\lambda_{0}(s)}, \ldots, P_{\lambda_{N-1}(s)}$ which are analytic over $\mathcal{I}$. Computing the derivative of

$$
H(s) P_{\lambda_{k}(s)}=\lambda_{k}(s) P_{\lambda_{k}(s)}
$$

with respect to $s$ at $s=\bar{s}$, we obtain

$$
H^{\prime}(\bar{s}) P_{\lambda_{k}(\bar{s})}+H(\bar{s}) P_{\lambda_{k}(\bar{s})}^{\prime}=\lambda_{k}^{\prime}(\bar{s}) P_{\lambda_{k}(\bar{s})}+\lambda_{k}(\bar{s}) P_{\lambda_{k}(\bar{s})}^{\prime} .
$$

Multiplying the last equation by $\left(P_{\lambda_{k}(\bar{s})}+P_{\lambda_{k+1}(\bar{s})}\right)$ from the left, using (22) and the fact that $P_{\lambda_{k}}$ and $P_{\lambda_{k+1}}$ are two orthogonal projectors $\left(P_{\lambda_{k}}^{2}=P_{\lambda_{k}}, P_{\lambda_{k+1}}^{2}=P_{\lambda_{k+1}}\right.$ and $\left.P_{\lambda_{k}} P_{\lambda_{k+1}}=0\right)$, we obtain $\left(P_{\lambda_{k}(\bar{s})}+P_{\lambda_{k+1}(\bar{s})}\right) H^{\prime}(\bar{s}) P_{\lambda_{k}(\bar{s})}=\lambda_{k}^{\prime}(\bar{s}) P_{\lambda_{k}(\bar{s})}$. Noting that $P_{\lambda_{k}(\bar{s})}=\left(P_{\lambda_{k}(\bar{s})}+P_{\lambda_{k+1}(\bar{s})}\right) P_{\lambda_{k}(\bar{s})}$, we obtain

$\left(P_{\lambda_{k}(\bar{s})}+P_{\lambda_{k+1}(\bar{s})}\right) H^{\prime}(\bar{s})\left(P_{\lambda_{k}(\bar{s})}+P_{\lambda_{k+1}(\bar{s})}\right) P_{\lambda_{k}(\bar{s})}=\lambda_{k}^{\prime}(\bar{s}) P_{\lambda_{k}(\bar{s})}$.

The analogue holds with $k$ and $k+1$ switched. This implies that $\left\{\lambda_{k}^{\prime}(\bar{s}), \lambda_{k+1}^{\prime}(\bar{s})\right\}$ are the eigenvalues of the $2 \times 2$ matrix obtained by restricting the operator $H^{\prime}(\bar{s})$ to the column space of $\left(P_{\lambda_{k}(\bar{s})}+P_{\lambda_{k+1}(\bar{s})}\right)$. Since $A(\bar{s})=0$ we have $H(\bar{s})=$ $H_{R}(\omega(\bar{s}))$. Denoting $|m\rangle$ and $|n\rangle$ the two eigenvectors of $H_{R}$ corresponding to the eigenvalue $\lambda_{k}(\bar{s})=\lambda_{k+1}(\bar{s})$, we have $P_{\lambda_{k}(\bar{s})}+P_{\lambda_{k+1}(\bar{s})}=|m\rangle\langle m|+| n\rangle\langle n|$. Defining

$$
\left(H^{\prime}(\bar{s})\right)_{m n}=\left(\begin{array}{ll}
\left\langle m\left|H^{\prime}(\bar{s})\right| m\right\rangle & \left\langle m\left|H^{\prime}(\bar{s})\right| n\right\rangle \\
\left\langle n\left|H^{\prime}(\bar{s})\right| m\right\rangle & \left\langle n\left|H^{\prime}(\bar{s})\right| n\right\rangle
\end{array}\right)
$$

and computing

$$
H^{\prime}(\bar{s})=\left.\omega^{\prime}(\bar{s}) \frac{\mathrm{d}}{\mathrm{d} v} H_{R}(v)\right|_{v=\omega(\bar{s})}+A^{\prime}(\bar{s}) H_{1} \text {, we obtain }
$$

$$
\left(H^{\prime}(\bar{s})\right)_{m n}=\left(\begin{array}{cc}
-m \omega^{\prime}(\bar{s}) & A^{\prime}(\bar{s}) \mu_{m n} \\
A^{\prime}(\bar{s}) \mu_{m n} & -n \omega^{\prime}(\bar{s})
\end{array}\right)
$$

where $\mu_{m n}=\left\langle m\left|H_{1}\right| n\right\rangle$. Thus $\mu_{m n}=0$ if $|m-n|>1$ and $\mu_{m n} \neq 0$ if $|m-n|=1$. In both cases, since $\omega^{\prime}(\bar{s}) \neq 0$ and $m \neq n$, the matrix in (23) has two real and distinct eigenvalues, corresponding to $\lambda_{k}^{\prime}(\bar{s}) \neq \lambda_{k+1}^{\prime}(\bar{s})$.

Proof (of theorem 2). Taking $A(s)=0$ at some points where $H_{R}$ is degenerate means that eigenvalues of $H(s)$ will not remain distinct at those points. We therefore use the adiabatic theorem without the spectral gap condition, see [13], corollary 2.5. Like for theorem 1 , we prove the result for one system $\in \mathcal{S}$ and conclude the result for the sup. The proof is again in two steps. First we state how the adiabatic theorem can be applied; then we compute the image at $s=1$ of the initial state $|k\rangle\langle k|$ in adiabatic approximation. 
Step 1: since $H$ is Hermitian, analytic over $[0,1]$ and simply degenerate at isolated points, we can apply lemma 2(a) repeatedly to conclude that there is a unique set of functions $\lambda_{0}, \ldots, \lambda_{N-1}$ analytic over $\mathcal{I}$, with $\lambda_{0}(0)<\cdots<\lambda_{N-1}(0)$, and such that $\left\{\lambda_{0}(s), \ldots, \lambda_{N-1}(s)\right\}$ are the eigenvalues of $H(s)$ for all $s \in \mathcal{I}$. Moreover, according to [30], section II.6.2, there is a unique set of associated rank-one projectors $P_{\lambda_{0}(s)}, \ldots, P_{\lambda_{N-1}(s)}$ which are analytic over $\mathcal{I}$. In particular, given assumption (A3) and as $H(s)=H_{R}(\omega(s))$ for $s \in$ $\{0,1\}$, we have $\left(\lambda_{k}(0), P_{\lambda_{k}(0)}\right)=\left(\lambda_{k}^{R}(0),|k\rangle\langle k|\right)$ for all $k$ and $\left\{\left(\lambda_{0}(1), P_{\lambda_{0}(1)}\right), \ldots,\left(\lambda_{N-1}(1), P_{\lambda_{N-1}(1)}\right)\right\}=$ $\left\{\left(\lambda_{0}^{R}(1),|0\rangle\langle 0|\right), \ldots,\left(\lambda_{N-1}^{R}(1),|N-1\rangle\langle N-1|\right)\right\}$. Note however that, unlike for theorem 1 , the pairwise correspondence between elements of the latter sets is not obvious a priori, because here eigenvalues of $H(s)$ do not remain distinct on $[0,1]$. A second difficulty is to assess how the system's state evolves when eigenvalues become degenerate. This second part is answered by the adiabatic theorem without gap condition. Introduce, as in theorem 1, the adiabatic Hamiltonian $H_{a, 0}$ and the adiabatic propagator $U_{a, 0}^{\varepsilon}$, given by (19) and (20) respectively with $k=0$. Then by construction $U_{a, 0}^{\varepsilon}(1)|0\rangle\langle 0| U_{a, 0}^{\varepsilon}(1)^{\dagger}=U_{a, 0}^{\varepsilon}(1) P_{\lambda_{0}(0)} U_{a, 0}^{\varepsilon}(1)^{\dagger}=P_{\lambda_{0}(1)}$. The adiabatic theorem states that $\exists C$ such that

$\| U^{\varepsilon}(s)|k\rangle\left\langle k\left|U^{\varepsilon}(s)^{\dagger}-U_{a, 0}^{\varepsilon}(s)\right| k\right\rangle\langle k| U_{a, 0}^{\varepsilon}(s)^{\dagger} \| \leqslant C \sqrt{\varepsilon}$

for all $|k\rangle \in\{|0\rangle, \ldots,|N-1\rangle\}$. Thus the actual system adiabatically follows the analytic $P_{\lambda_{k}(s)}$, from $P_{\lambda_{0}(0)}=$ $|0\rangle\langle 0|$ up to $P_{\lambda_{0}(1)}$ in particular.

Step 2: we now compute $P_{\lambda_{0}(1)}$. Define a small interval $I_{m n}=\left[\tau_{m n}^{o}, \tau_{m n}^{f}\right] \subset[0,1]$ around each point $s(m, n)$ such that all $I_{m n}$ are disjoint. If $A(s(m, n)) \neq 0$, then, as shown in theorem $1, H(s)$ is non-degenerate for all $s \in I_{m n}$, such that for any $j, k$ with $\lambda_{j}\left(\tau_{m n}^{o}\right)<\lambda_{k}\left(\tau_{m n}^{o}\right)$ we have $\lambda_{j}\left(\tau_{m n}^{f}\right)<\lambda_{k}\left(\tau_{m n}^{f}\right)$. On the other hand, if we take $A(s(m, n))=0$, then two eigenvalues intersect at $s=s(m, n)$ and the analytic branches cross so that their order changes as stated in lemma 2(b). To avoid separate treatment of limit cases, we define $s_{0}=0$ and $s_{N}=1$. Now by construction:

- $\lambda_{j}(0)=\lambda_{j}^{R}(0)$ for all $j \in \mathbb{N}_{0}^{N-1}$.

- For $k \in \mathbb{N}_{1}^{N}, \lambda_{0}^{R}(s)$ is the $k$ th smallest eigenvalue of $H_{R}(\omega(s))$ when $s \in\left(s_{k-1}, s_{k}\right)$.

- As long as $A\left(s_{0}\right)=\cdots=A\left(s_{k-1}\right)=0$, that is for $k \leqslant N-p, \lambda_{0}(s)$ follows the same crossings as $\lambda_{0}^{R}(s)$; therefore it is the $k$ th smallest eigenvalue of $H(s)$ when $s \in\left(s_{k-1}, s_{k}\right)$.

- For $s>s_{N-p-1}$, we have $A(s) \neq 0$ so the $\lambda_{k}(s)$ keep the same order, i.e. $\lambda_{0}(s)$ remains the $(N-p)$ th smallest eigenvalue of $H(s)$.

- In particular for $s=1$, from (13) we identify $\lambda_{0}(1)=$ $\lambda_{N-(N-p)}^{R}(1)=\lambda_{p}^{R}(1)$, such that $P_{\lambda_{0}(1)}=|p\rangle\langle p|$ by uniqueness of the spectral decomposition.
Remark 2. To apply the adiabatic theorem [13], corollary 2.5, it is sufficient to have $P_{\lambda_{0}(s)} \in \mathcal{C}^{2}\left([0,1], \mathcal{H}_{N}\right)$. However, a condition like $H(s) \in \mathcal{C}^{2}\left([0,1], \mathcal{H}_{N}\right)$ does not ensure the existence of $P_{\lambda_{0}(s)}, \ldots, P_{\lambda_{N-1}(s)} \in \mathcal{C}^{2}\left([0,1], \mathcal{H}_{N}\right)$, see [30], example 5.3. It is only for analytic $H(s)$ that we can guarantee analytic $P_{\lambda_{0}(s)}$, which then in particular belongs to $\mathcal{C}^{2}\left([0,1], \mathcal{H}_{N}\right)$.

Proof (of corollary 1). The arguments are the same as in the proof of theorem 2. We concentrate on tracking the analytic eigenvalue branches $\lambda_{0}(s), \ldots, \lambda_{N-1}(s)$ of $H(s)$ to establish their pairwise correspondence with eigenvalues $\lambda_{0}^{R}(s), \ldots, \lambda_{N-1}^{R}(s)$ of $H_{R}(\omega(s))$ at $s=1$. We prove the result for $p<N-l-1$; the case $p>N-l-1$ is treated similarly, while $p=N-l-1$, implying $\mathcal{I}_{l p}^{\omega}=\emptyset$, is the case covered by theorem 1 . Denote by $s_{1}<\cdots<s_{N-l-p-1}$ the elements of $\mathcal{I}_{l p}^{\omega}$, and $s_{0}=0, s_{N-l-p}=1$.

The algorithm constructs $\mathcal{I}_{l p}^{\omega}$ such that the $(l+d)$ th and $(l+d+1)$ th smallest eigenvalues of $H_{R}(\omega(s))$ become equal at $s_{d}$, for each $d \in \mathbb{N}_{1}^{N-l-p-1}$. Taking $A\left(s_{d}\right)=0$ implies $H\left(s_{d}\right)=H_{R}\left(\omega\left(s_{d}\right)\right)$ so the same eigenvalue equalities hold for $H(s)$ at $s=s_{d}$. Moreover from point (c) and lemma 1 all eigenvalues of $H(s)$ remain distinct for $s \notin \mathcal{I}_{l p}^{\omega}$. Therefore the analytic eigenvalue branch $\lambda_{l}(s)$, starting with $\lambda_{l}(0)=\lambda_{l}^{R}(0)$, exactly evolves through crossings at $s_{1}, \ldots, s_{N-l-p-1}$ such that it is the $(l+d+1)$ th smallest eigenvalue of $H(s)$ for $s \in\left(s_{d}, s_{d+1}\right)$. In particular, $\lambda_{l}(1)$ is the $(N-p)$ th smallest eigenvalue of $H(1)=H_{R}(\omega(1))$, which from (A2) means $\lambda_{l}(1)=\lambda_{p}^{R}(1)$ such that $P_{\lambda_{l}(1)}=|p\rangle\langle p|$.

\section{Summary and discussion}

This paper shows how adiabatic passage can be applied to a quantum ladder system to achieve permutations of populations on the ladder levels with a single laser pulse. We explicitly propose control inputs whose precise functional dependence on time is not important as long as they satisfy a few key features, most notably annihilation or not at specific times. This makes our strategy robust against multiplicative input disturbances. Another important advantage of our adiabatic strategy is its ability to simultaneously control an ensemble of systems with different dipole moment values.

Theorems in this paper provide a proof of concept in idealized situations. Several practical issues deserve a more quantitative investigation in future work. Probably the most important aspect is to characterize precision of the adiabatic approximation as a function of $\varepsilon$. Indeed, for small $\varepsilon$ the actual control time $t=\frac{s}{\varepsilon}$ gets long; this further implies that, at a constant power $A^{2}(s)$, the energy given to the system gets large. Beyond performance requirements, this also invalidates our model at infinitesimal $\varepsilon$ (e.g. regarding finite lifetime of the levels). Although orders of magnitude are given for the adiabatic limit, variations in the proportionality constant can lead to significant discrepancies. Investigating them, as well as 'optimal paths' minimizing non-adiabatic losses [31], could yield guidelines for choosing amongst several possible 'eigenvalue crossing designs'. Both precision of adiabatic 
approximation and modelling assumptions (e.g. RWA) also limit the range of 'ensemble' properties in practice.

It may appear surprising at first sight that two different evolutions are selected just by taking $A(\bar{s})=0$ or $A(\bar{s}) \neq 0$ at a precise instant $\bar{s}$. The elucidation is that this dichotomy only holds at the limit $\varepsilon \rightarrow 0^{+}$. For a given $\varepsilon$, the larger the $|A|$ in the neighbourhood of $s=\bar{s}$, the more the evolution differentiates from the $A(\bar{s})=0$ case. Nevertheless, for small $\varepsilon$, the relevant neighbourhood around $\bar{s}$ for selecting population transfer or not indeed gets small (from there experimentalists' denomination 'rapid adiabatic passage'). Our scheme might therefore allow selective population permutation as a function of $\left\{\Delta_{0}, \ldots, \Delta_{N-1}\right\}$ on an ensemble of systems, in a scheme resembling resonance selection. Take $A(\bar{s})=0$ for $\bar{s} \in \mathcal{I}^{\omega}$ of a nominal system. If a system has detunings very close to nominal, then two of its $\lambda_{k}^{R}(s)$ cross at a point $\tilde{s}$ close to $\bar{s}$, where $A(\widetilde{s}) \approx 0$, such that for moderate $\varepsilon$ its final state will be close to the adiabatic result of the nominal system with $A(\bar{s})=0$. If a system has detunings more different from nominal, then all its crossings of $\lambda_{k}^{R}(s)$ occur at points where $A$ significantly differs from zero, and with moderate $\varepsilon$ its final state will be closer to the adiabatic result of the nominal system with $A(\bar{s}) \neq 0$. A quantitative statement of this idea requires further investigation.

\section{Acknowledgments}

The authors thank M Lemaire for useful discussions. ZL acknowledges support from ANR, Projet Jeunes Chercheurs EPOQ2 number ANR-09-JCJC-0070. AS is an FNRS postdoctoral researcher. This paper presents research results of the Belgian Network DYSCO (Dynamical Systems, Control, and Optimization), funded by the Interuniversity Attraction Poles Programme, initiated by the Belgian State, Science Policy Office. The scientific responsibility rests with its authors. PR acknowledges support from ANR (CQUID).

\section{References}

[1] Nussenzweig P, Bernardot F, Brune M, Hare J, Raimond J M, Haroche S and Gawlik W 1993 Preparation of high-principal-quantum-number 'circular' states of rubidium Phys. Rev. A 48 3991-3995

[2] Nielsen M A and Chuang I L 2000 Quantum Computation and Quantum Information (Cambridge: Cambridge University Press)

[3] Averin D V 1998 Adiabatic quantum computation with cooper pairs Solid State Commun. 105 659-64

[4] Aharonov D, van Dam W, Kempe J, Landau Z, Lloyd S and Regev O 2007 Adiabatic quantum computation is equivalent to standard quantum computation SIAM J. Comput. 37 166-94

[5] Chelkowski S, Bandrauk A D and Corkum P B 1990 Efficient molecular dissociation by a chirped ultrashort infrared laser pulse Phys. Rev. Lett. 65 2355-8

[6] Yatsenko L P, Guérin S and Jauslin H R 2002 Topology of adiabatic passage Phys. Rev. A $\mathbf{6 5} 043407$
[7] Boscain U, Chittaro F C, Mason P and Sigalotti M 2011 Adiabatric control of the Schrödinger equation via conical intersection of eigenvalues IEEE Trans. Automatic Control submitted

[8] Li J-S and Khaneja N 2009 Ensemble control of Bloch equations IEEE Trans. Autom. Control 54 528-36

[9] Li J-S and Khaneja N 2006 Control of inhomogeneous quantum ensembles Phys. Rev. A 73030302

[10] Beauchard K, Coron J M and Rouchon P 2010 Controllability issues for continuous-spectrum systems and ensemble controllability of Bloch equations Commun. Math. Phys. $296525-57$

[11] Avron J E, Seiler R and Yaffe L G 1987 Adiabatic theorems and applications to the quantum hall effect Commun. Math. Phys. $11033-49$

[12] Avron J E and Elgart A 1999 Adiabatic theorem without a gap condition Commun. Math. Phys. 203 445-63

[13] Teufel S 2003 Adiabatic Perturbation Theory in Quantum Dynamics (Berlin: Springer)

[14] Messiah A 1958 Quantum Mechanics (New York: Wiley)

[15] Allen L and Eberly J H 1987 Optical Resonance and Two-Level Atoms (New York: Dover)

[16] Oreg J, Hioe F T and Eberly J H 1984 Adiabatic following in multilevel systems Phys. Rev. A 29 690-697

[17] Shapiro E A, Milner V and Shapiro M 2009 Complete transfer of populations from a single state to a preselected superposition of states using piecewise adiabatic passage: theory Phys. Rev. A 79023422

[18] Vitanov N V, Halfmann T, Shore B W and Bergmann K 2001 Laser-induced population transfer by adiabatic passage techniques: a review Annu. Rev. Phys. Chem. 52 763-809

[19] Hulet R G and Kleppner D 1983 Rydberg atoms in 'circular' states Phys. Rev. Lett. 51 1430-3

[20] Maas D J, Rella C W, Antoine P, Toma E S and Noordam L D 1999 Population transfer via adiabatic passage in the rubidium quantum ladder system Phys. Rev. A 59 1374-1381

[21] Guérin S 1997 Complete dissociation by chirped laser pulses designed by adiabatic Floquet analysis Phys. Rev. A 56 1458-1462

[22] Favard J 1935 Sur les polynomes de Tchebicheff C.R. Acad. Sci. Paris $2002052-3$

[23] Szegö G 1967 Orthogonal Polynomials (Providence, RI: American Mathematical Society)

[24] Boscain U, Chittaro F C, Mason P, Pacqueau R and Sigalotti M 2010 Motion planning in quantum control via intersection of eigenvalues Proc. 49th IEEE Conf. Decision and Control pp 3028-33

[25] Weiner A M 2000 Femtosecond pulse shaping using spatial light modulators Rev. Sci. Instrum. 71 1929-60

[26] Sanders J, Verhulst F and Murdock J 2007 Averaging Methods in Nonlinear Dynamical Systems (New York: Springer)

[27] Thomas S, Guérin S and Jauslin H R 2005 State-selective chirped adiabatic passage on dynamically laser-aligned molecules Phys. Rev. A 71013402

[28] Nagao T and Wadati M 1993 Level statistics of discrete Schrödinger equations and orthogonal polynomials J. Phys. Soc. Japan 62 46-52

[29] Khalil H K 2001 Nonlinear Systems (Englewood Cliffs, NJ: Prentice Hall)

[30] Kato T 1966 Perturbation Theory for Linear Operators (Berlin: Springer)

[31] Guérin S, Thomas S and Jauslin H R 2002 Optimization of population transfer by adiabatic passage Phys. Rev. A 65023409 